\title{
Caracterización física y mecánica de compuestos de Guazuma crinita Mart. a base de polipropileno virgen
} Physical and mechanical characterization of Guazuma
crinita Mart. composites based on virgin polypropylene

\author{
Alexei Rodolfo Córdova Contreras ${ }^{1}$ Aldo Joao Cárdenas Oscanoa ${ }^{2 *}$ \\ y Héctor Enrique Gonzáles Mora ${ }^{2}$
}

\begin{abstract}
Wood plastic composite materials based on Guazuma crinita wood particles (White Bolaina) from forestry thinning of 4, 5 and 6 years old and virgin polypropylene (PP) were prepared, using polypropylene maleic anhydride (MAPP) as a coupling agent. Specimens were made by extrusion, thermal compression and laser cutting. White bolaine particles were sieved with ASTM mesh sizes: $-40 /+60,-60 /+80$ and $-80 /+100$. Proportions of polypropylene/Bolaina mixture were: 70/30, 80/20 and 90/10. All formulations included $2 \%$ MAPP as a coupling agent. Physical properties as moisture content, density, absorption and swelling were assessed, as well as the mechanical properties of static bending, tension and impact resistance. Additionally, for discussing the results an anatomical characterization of the White Bolaina wood fiber (length, diameter, wall thickness, lumen diameter and slenderness coefficient) and a chemical analysis of its components (extractives, holocellulose, lignin and ash) was carried out. Results allow to appreciate a direct relationship between the variable mixing ratio and the mainly physical properties, as well as between the variable particle size with respect to tension. Wood age did not represent a significant source of variability.
\end{abstract}

Key words: Guazuma crinita Mart., wood plastic composites, modulus of elasticity, polypropylene, physical properties, mechanical properties.

\section{Resumen}

Se elaboraron materiales compuestos plástico madera con base en partículas de madera de la especie Guazuma crinita (bolaina blanca), proveniente del raleo de 4, 5 y 6 años, con polipropileno virgen (PP), además se utilizó como agente acoplante anhídrido maleíco de polipropileno (MAPP). La producción de probetas se hizo por el método de extrusión, compresión térmica y corte por láser. Las partículas de bolaina blanca se tamizaron con tamaños de malla ASTM: $-40 /+60,-60 /+80$ y $-80 /+100$. Las proporciones de mezcla polipropileno/bolaina fueron: 70/30, 80/20 y 90/10. Todas las formulaciones incluyeron $2 \%$ de MAPP como agente acoplante. Se evaluaron las propiedades físicas de contenido de humedad, densidad, absorción e hinchamiento; así como, las propiedades mecánicas de flexión estática, tensión y resistencia al impacto. Para la discusión de resultados se realizó una caracterización anatómica de la fibra (longitud, diámetro, espesor de pared, diámetro del lumen y coeficiente de esbeltez), además de un análisis químico de los componentes de la misma (extractivos, holocelulosa, lignina y cenizas). Los resultados permiten apreciar influencia de la variable proporción de mezcla sobre las principales propiedades físicas, así como de la variable tamaño de partícula respecto a la tensión. La edad de la madera no representó una fuente de variabilidad significativa.

Palabras claves: Guazuma crinita Mart., materiales compuestos plástico madera, módulo de elasticidad, polipropileno, propiedades físicas, propiedades mecánicas.

\footnotetext{
${ }^{1}$ Universidad Nacional Agraria La Molina. Perú

${ }^{2}$ Departamento de Industrias Forestales, Facultad de Ciencias Forestales, Universidad Nacional Agraria La Molina. Perú

*Autor para correspondencia; correo-e: aldo.cardenas@gmail.com
} 


\section{Introducción}

A lo largo de la historia, se han propuesto muchas fibras lignocelulósicas como refuerzo en materiales compuestos. De especial interés resulta la utilización de fibras de madera de rápido crecimiento, por ser un recurso natural de bajo costo, renovable y de gran disponibilidad para fines industriales; así como por sus aportes en las características físicas y mecánicas a los productos finales (Satyanarayana et al., 2009).

A esto se suma la demanda de la sociedad por productos originados a partir de residuos industriales y de un solo uso, como el aserrín y el plástico, respectivamente. Todo ello ha permitido que sectores de las industrias de la construcción y automotriz desarrollen una variedad de productos, entre ellos barandillas, marcos de ventanas, paneles de puertas, molduras, pisos, tapicería de asientos, etcétera (Clemons, 2002).

En los materiales compuestos se busca el tamaño óptimo de las partículas, así como su adecuada proporción, de acuerdo a su uso final. Los materiales compuestos reforzados con fibras vegetales han estado posicionándose con fuerza en el mercado, favorecidos por su bajo costo, alta durabilidad y la utilización de residuos en su elaboración (Wolcott y Englund, 1999; Klyosov, 2007).

En Perú existen pocos antecedentes de investigaciones relacionadas con materiales compuestos de plástico y madera (Lázaro et al., 2016a; Lázaro et al., 2016b; Gonzáles et al., 2018), a pesar de los esfuerzos realizados por las universidades, organismos del Estado y entidades privadas para su promoción; solo hay una empresa nacional que los produce de plástico y madera y los comercializa. Su principal mercado son las empresas constructoras que las utilizan para proyectos multifamiliares, por su bajo costo de instalación y mantenimiento, así como por su prolongado tiempo de servicio, característica valiosa para este fin.

Es importante la definición de la materia prima que se va a utilizar, pues debe proceder de una especie promisoria industrialmente y con plantaciones ya existentes. Tal es el caso de la Bolaina blanca (Guazuma crinita Mart.), especie forestal muy difundida como alternativa para plantaciones forestales en la Amazonia peruana. El principal producto de la Bolaina blanca es la madera aserrada, con la cual se fabrican machihembrados para interiores y exteriores, 
tableros encolados, molduras, interior de muebles y otros tipos de carpintería (Guerra et al., 2008).

Con base en lo anterior, en el presente estudio se planteó como objetivo hacer una caracterización de materiales compuestos a base de polipropileno virgen, reforzados con partículas de Bolaina blanca, mediante la evaluación de las propiedades físicas y mecánicas.

\section{Materiales y Métodos}

Se elaboraron materiales compuestos plástico madera, para los cuales, como material de refuerzo, se utilizó madera de raleo de 4, 5 y 6 años de Guazuma crinita (bolaina blanca), procedente de una plantación forestal ubicada en la provincia de Puerto Inca, departamento de Huánuco, Perú.

Como matriz termoplástica se usó un homopolímero de polipropileno, con índice

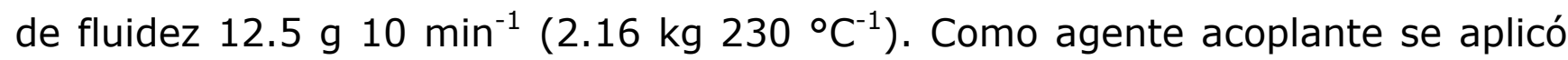
anhídrido maleico de polipropileno (MAPP), sobre el cual existen diversos estudios de eficiencia (Correa et al., 2007), con una temperatura de fusión de $167^{\circ} \mathrm{C}$; en concentración de 2 \% para todas las formulaciones.

La madera se dejó secar, se descortezó, molió y tamizó hasta producir partículas de tres tamaños de malla (ASTM): -40/+60, $-60 /+80$ y $-80 /+100$. Posteriormente, se secaron en estufa a $103{ }^{\circ} \mathrm{C} \pm 2{ }^{\circ} \mathrm{C}$ por $48 \mathrm{~h}$ para alcanzar un contenido de humedad $<5 \%$. Se elaboraron las diferentes formulaciones propuestas (Cuadro 1). La extrusión de los materiales se efectuó en una máquina de husillo simple, a una temperatura entre $160-175^{\circ} \mathrm{C}$ y $30 \mathrm{rpm}$; enseguida, el material extrusado se molió para continuar con el prensado. 
Cuadro 1. Formulaciones con diferentes edades, tamaños de partícula, proporciones y tratamiento.

\begin{tabular}{|c|c|c|c|c|c|}
\hline \multirow{2}{*}{$\begin{array}{c}\text { Edad } \\
\text { (años) }\end{array}$} & \multirow{2}{*}{$\begin{array}{c}\text { Tamaño } \\
\text { de malla } \\
\text { ASTM }\end{array}$} & \multirow{2}{*}{$\begin{array}{c}\text { Tamaño } \\
\text { de } \\
\text { partícula } \\
(\mu \mathrm{m})\end{array}$} & \multicolumn{2}{|c|}{ Composición } & \multirow{2}{*}{$\begin{array}{l}\text { Código de } \\
\text { tratamiento }\end{array}$} \\
\hline & & & $\begin{array}{c}\text { Bolaina } \\
(\%)\end{array}$ & $\begin{array}{c}\text { Polipropileno } \\
(\%)\end{array}$ & \\
\hline \multirow{9}{*}{4} & \multirow{3}{*}{$+40 /-60$} & \multirow{3}{*}{$400-250$} & 30 & 70 & $\mathrm{~T}-4 / 40 / 30$ \\
\hline & & & 20 & 80 & $T-4 / 40 / 20$ \\
\hline & & & 10 & 90 & $\mathrm{~T}-4 / 40 / 10$ \\
\hline & \multirow{3}{*}{$+60 /-80$} & \multirow{3}{*}{$250-177$} & 30 & 70 & $T-4 / 60 / 30$ \\
\hline & & & 20 & 80 & $T-4 / 60 / 20$ \\
\hline & & & 10 & 90 & $\mathrm{~T}-4 / 60 / 10$ \\
\hline & \multirow{3}{*}{$+80 /-100$} & \multirow{3}{*}{$177-149$} & 30 & 70 & $\mathrm{~T}-4 / 80 / 30$ \\
\hline & & & 20 & 80 & $\mathrm{~T}-4 / 80 / 20$ \\
\hline & & & 10 & 90 & $\mathrm{~T}-4 / 80 / 10$ \\
\hline \multirow{9}{*}{5} & \multirow{3}{*}{$+40 /-60$} & \multirow{3}{*}{$400-250$} & 30 & 70 & T-5/40/30 \\
\hline & & & 20 & 80 & $T-5 / 40 / 20$ \\
\hline & & & 10 & 90 & $\mathrm{~T}-5 / 40 / 10$ \\
\hline & \multirow{3}{*}{$+60 /-80$} & \multirow{3}{*}{$250-177$} & 30 & 70 & T-5/60/30 \\
\hline & & & 20 & 80 & $T-5 / 60 / 20$ \\
\hline & & & 10 & 90 & $\mathrm{~T}-5 / 60 / 10$ \\
\hline & \multirow{3}{*}{$+80 /-100$} & \multirow{3}{*}{$177-149$} & 30 & 70 & T-5/80/30 \\
\hline & & & 20 & 80 & $\mathrm{~T}-5 / 80 / 20$ \\
\hline & & & 10 & 90 & $\mathrm{~T}-5 / 80 / 10$ \\
\hline \multirow{9}{*}{6} & \multirow{3}{*}{$+40 /-60$} & \multirow{3}{*}{$400-250$} & 30 & 70 & T-6/40/30 \\
\hline & & & 20 & 80 & T-6/40/20 \\
\hline & & & 10 & 90 & $\mathrm{~T}-6 / 40 / 10$ \\
\hline & \multirow{3}{*}{$+60 /-80$} & \multirow{3}{*}{$250-177$} & 30 & 70 & T-6/60/30 \\
\hline & & & 20 & 80 & T-6/60/20 \\
\hline & & & 10 & 90 & T-6/60/10 \\
\hline & \multirow{3}{*}{$+80 /-100$} & \multirow{3}{*}{$177-149$} & 30 & 70 & T-6/80/30 \\
\hline & & & 20 & 80 & T-6/80/20 \\
\hline & & & 10 & 90 & T-6/80/10 \\
\hline
\end{tabular}


Los tableros de material compuesto se hicieron en una prensa hidráulica, a una velocidad de $0.9 \mathrm{~cm} \mathrm{~s}^{-1}$ y presión de 40 bares; el curado del material tardó de 4 a 5 minutos, a una temperatura entre 177 y $195^{\circ} \mathrm{C}$. Con una máquina láser de 80 W de potencia se elaboraron 1080 probetas. Se emplearon 40 por cada tratamiento de acuerdo a las siguientes normas: ASTM D1037-99 para humedad y densidad (ASTM, 1999), ASTM D570-98 para absorción e hinchamiento (ASTM, 1998), ASTM D638-03 para tensión (ASTM, 2003b), ASTM D790-03 para flexión estática (ASTM, 2003a) y ASTM D5420-04 para resistencia al impacto (ASTM, 2004). El modelo estadístico del diseño factorial utilizado fue:

$$
X_{i j k}=\mu+E_{i}+T_{j}+P_{k}+(E T)_{i j}+(E P)_{i k}+(T P)_{j k}+(E T P)_{i j k}+\varepsilon
$$

Donde:

$X_{i j k}=$ Observación correspondiente a la i-ésima repetición

$\mu=$ Media de todas las observaciones del tratamiento

$E_{i}=$ Parámetro que mide el efecto de la variable principal edad

$T_{j}=$ Parámetro que mide el efecto de la variable principal tamaño de partícula

$P_{k}=$ Parámetro que mide el efecto de la variable principal proporción de mezcla

$(E T)_{i j}=$ Efecto de la interacción doble de las variables edad y tamaño de partícula

$(E P)_{i k}=$ Efecto de la interacción doble de las variables edad y proporción de mezcla $(T P)_{j k}=$ Efecto de la interacción doble de las variables tamaño de partícula y proporción de mezcla

$(E T P)_{i j k}=$ Efecto de la interacción triple de las variables edad, tamaño de las variables partícula y proporción de mezcla

$\varepsilon=$ Error experimental

Se aplicó un análisis de varianza con el programa Statistical Analysis System versión 9.1 (SAS), con intervalo de confianza de $95 \%$. 
La caracterización anatómica de las fibras de bolaina blanca se realizó de acuerdo con la norma de procedimientos para estudios de anatomía de la madera (Ibama, 1991). Se consideraron los valores de longitud, ancho y espesor de la pared de al menos 25 haces fibrosos procedentes del proceso de desfibrado, con una cámara LEICA ICC50 HD acoplada a un microscopio LEICA DM500 con aumentos de 4X, 10X y 40X.

La caracterización química de las fibras se hizo con base en las normas: TAPPI T 264 CM-97 (TAPPI; 1997a) para preparación de madera en análisis químico, TAPPI T 204 CM-97 (TAPPI; 1997b) para extractivos, método Jayme-Wise para holocelulosa, TAPPI T 222 OM-98 (TAPPI; 1998) para lignina insoluble, y TAPPI T 211 OM-93 para cenizas (TAPPI, 1993).

\section{Resultados y Discusión}

Se evaluaron las propiedades físicas y mecánicas del material compuesto PP-Bolaina blanca (Cuadro 2), además de una caracterización de la fibra (Cuadro 3) y una descripción química de la misma en sus tres edades. 
Cuadro 2. Valores promedio y desviación estándar de las propiedades físicas y mecánicas del material compuesto PP-Bolaina blanca de 4, 5 y 6 años de edad.

\begin{tabular}{|c|c|c|c|c|c|c|c|c|c|}
\hline \multirow[b]{2}{*}{ Tratamiento } & \multirow{2}{*}{$\begin{array}{c}\text { Contenido } \\
\text { de } \\
\text { humedad } \\
(\%)\end{array}$} & \multirow[b]{2}{*}{$\begin{array}{l}\text { Densidad } \\
\left(\mathrm{g} \mathrm{cm}^{-3}\right)\end{array}$} & \multirow[b]{2}{*}{$\begin{array}{c}\text { Absorción } \\
(\%)\end{array}$} & \multirow[b]{2}{*}{$\begin{array}{c}\text { Hinchamiento } \\
(\%)\end{array}$} & \multicolumn{2}{|c|}{ Tensión } & \multicolumn{2}{|c|}{ Flexión estática } & \multirow[b]{2}{*}{$\begin{array}{c}\text { Impacto } \\
\text { (J) }\end{array}$} \\
\hline & & & & & $\begin{array}{c}\text { Resistencia } \\
\text { máxima } \\
(\mathrm{MPa})\end{array}$ & $\begin{array}{c}\text { Módulo de } \\
\text { elasticidad } \\
\text { (Gpa) }\end{array}$ & $\begin{array}{c}\text { Resistencia } \\
\text { máxima } \\
\text { (MPa) }\end{array}$ & $\begin{array}{c}\text { Módulo de } \\
\text { elasticidad } \\
\text { (Gpa) }\end{array}$ & \\
\hline $\mathrm{T}-4 / 40 / 20$ & $\begin{array}{c}1.5 \\
{[0.13]}\end{array}$ & $\begin{array}{c}0.87 \\
{[0.02]}\end{array}$ & $\begin{array}{c}7.7 \\
{[1.63]}\end{array}$ & $\begin{array}{c}4.0 \\
{[1.63]}\end{array}$ & $\begin{array}{c}16.7 \\
{[0.43]}\end{array}$ & $\begin{array}{c}0.9 \\
{[0.21]}\end{array}$ & $\begin{array}{c}30.4 \\
{[2.04]}\end{array}$ & $\begin{array}{c}1.1 \\
{[0.14]}\end{array}$ & $\begin{array}{c}0.62 \\
{[0.05]}\end{array}$ \\
\hline $\mathrm{T}-4 / 40 / 10$ & $\begin{array}{c}1.0 \\
{[0.06]}\end{array}$ & $\begin{array}{c}0.76 \\
{[0.06]}\end{array}$ & $\begin{array}{c}17.4 \\
{[6.60]}\end{array}$ & $\begin{array}{c}3.2 \\
{[1.45]}\end{array}$ & $\begin{array}{c}16.7 \\
{[2.12]}\end{array}$ & $\begin{array}{c}0.9 \\
{[0.15]}\end{array}$ & $\begin{array}{c}28.8 \\
{[2.34]}\end{array}$ & $\begin{array}{c}0.9 \\
{[0.08]}\end{array}$ & $\begin{array}{c}0.50 \\
{[0.05]}\end{array}$ \\
\hline$T-4 / 60 / 30$ & $\begin{array}{c}2.2 \\
{[0.07]}\end{array}$ & $\begin{array}{c}0.91 \\
{[0.01]}\end{array}$ & $\begin{array}{c}11.9 \\
{[0.83]}\end{array}$ & $\begin{array}{c}4.2 \\
{[0.56]}\end{array}$ & $\begin{array}{c}11.6 \\
{[1.12]}\end{array}$ & $\begin{array}{c}0.7 \\
{[0.08]}\end{array}$ & $\begin{array}{c}26.2 \\
{[4.28]}\end{array}$ & $\begin{array}{c}1.2 \\
{[0.11]}\end{array}$ & $\begin{array}{c}0.58 \\
{[0.07]}\end{array}$ \\
\hline $\mathrm{T}-4 / 60 / 20$ & $\begin{array}{c}1.5 \\
{[0.04]}\end{array}$ & $\begin{array}{c}0.87 \\
{[0.01]}\end{array}$ & $\begin{array}{c}8.2 \\
{[1.32]}\end{array}$ & $\begin{array}{c}3.3 \\
{[0.98]}\end{array}$ & $\begin{array}{c}12.9 \\
{[1.12]}\end{array}$ & $\begin{array}{c}0.6 \\
{[0.06]}\end{array}$ & $\begin{array}{c}28.8 \\
{[1.71]}\end{array}$ & $\begin{array}{c}1.1 \\
{[0.09]}\end{array}$ & $\begin{array}{c}0.48 \\
{[0.09]}\end{array}$ \\
\hline $\mathrm{T}-4 / 60 / 10$ & $\begin{array}{c}0.9 \\
{[0.10]}\end{array}$ & $\begin{array}{c}0.81 \\
{[0.03]}\end{array}$ & $\begin{array}{c}9.3 \\
{[5.98]}\end{array}$ & $\begin{array}{c}3.7 \\
{[0.23]}\end{array}$ & $\begin{array}{c}15.5 \\
{[1.05]}\end{array}$ & $\begin{array}{c}0.5 \\
{[0.04]}\end{array}$ & $\begin{array}{c}33.7 \\
{[1.00]}\end{array}$ & $\begin{array}{c}1.1 \\
{[0.04]}\end{array}$ & $\begin{array}{c}0.46 \\
{[0.01]}\end{array}$ \\
\hline $\mathrm{T}-4 / 80 / 30$ & $\begin{array}{c}2.2 \\
{[0.09]}\end{array}$ & $\begin{array}{c}0.91 \\
{[0.02]}\end{array}$ & $\begin{array}{c}12.2 \\
{[1.79]}\end{array}$ & $\begin{array}{c}5.8 \\
{[1.09]}\end{array}$ & $\begin{array}{c}13.9 \\
{[1.47]}\end{array}$ & $\begin{array}{c}0.7 \\
{[0.17]}\end{array}$ & $\begin{array}{c}27.4 \\
{[0.77]}\end{array}$ & $\begin{array}{c}1.2 \\
{[0.05]}\end{array}$ & $\begin{array}{c}0.56 \\
{[0.04]}\end{array}$ \\
\hline $\mathrm{T}-4 / 80 / 20$ & $\begin{array}{c}1.6 \\
{[0.11]}\end{array}$ & $\begin{array}{c}0.87 \\
{[0.01]}\end{array}$ & $\begin{array}{c}6.9 \\
{[0.92]}\end{array}$ & $\begin{array}{c}3.1 \\
{[0.71]}\end{array}$ & $\begin{array}{c}13.7 \\
{[0.77]}\end{array}$ & $\begin{array}{c}0.6 \\
{[0.03]}\end{array}$ & $\begin{array}{c}30.6 \\
{[1.97]}\end{array}$ & $\begin{array}{c}1.2 \\
{[0.08]}\end{array}$ & $\begin{array}{c}0.54 \\
{[0.05]}\end{array}$ \\
\hline $\mathrm{T}-4 / 80 / 10$ & $\begin{array}{c}2.0 \\
{[0.05]}\end{array}$ & $\begin{array}{c}0.83 \\
{[0.04]}\end{array}$ & $\begin{array}{c}5.7 \\
{[1.75]}\end{array}$ & $\begin{array}{c}3.7 \\
{[0.50]}\end{array}$ & $\begin{array}{c}15.7 \\
{[1.30]}\end{array}$ & $\begin{array}{c}0.6 \\
{[0.14]}\end{array}$ & $\begin{array}{c}31.4 \\
{[2.24]}\end{array}$ & $\begin{array}{c}1.1 \\
{[0.10]}\end{array}$ & $\begin{array}{c}0.49 \\
{[0.03]}\end{array}$ \\
\hline$T-5 / 40 / 30$ & $\begin{array}{c}2.7 \\
{[0.32]}\end{array}$ & $\begin{array}{c}0.91 \\
{[0.01]}\end{array}$ & $\begin{array}{c}16.3 \\
{[3.66]}\end{array}$ & $\begin{array}{c}5.8 \\
{[0.85]}\end{array}$ & $\begin{array}{c}13.2 \\
{[0.98]}\end{array}$ & $\begin{array}{c}0.8 \\
{[0.05]}\end{array}$ & $\begin{array}{c}29.8 \\
{[2.34]}\end{array}$ & $\begin{array}{c}1.1 \\
{[0.13]}\end{array}$ & $\begin{array}{c}0.45 \\
{[0.03]}\end{array}$ \\
\hline$T-5 / 40 / 20$ & $\begin{array}{c}1.9 \\
{[0.48]}\end{array}$ & $\begin{array}{c}0.89 \\
{[0.02]}\end{array}$ & $\begin{array}{c}18.8 \\
{[4.27]}\end{array}$ & $\begin{array}{c}4.2 \\
{[0.62]}\end{array}$ & $\begin{array}{c}13.3 \\
{[0.63]}\end{array}$ & $\begin{array}{c}0.8 \\
{[0.13]}\end{array}$ & $\begin{array}{c}28.3 \\
{[1.27]}\end{array}$ & $\begin{array}{c}1.0 \\
{[0.07]}\end{array}$ & $\begin{array}{c}0.45 \\
{[0.05]}\end{array}$ \\
\hline $\mathrm{T}-5 / 40 / 10$ & $\begin{array}{c}1.7 \\
{[0.33]}\end{array}$ & $\begin{array}{c}0.85 \\
{[0.02]}\end{array}$ & $\begin{array}{c}6.7 \\
{[2.81]}\end{array}$ & $\begin{array}{c}4.6 \\
{[1.04]}\end{array}$ & $\begin{array}{c}16.2 \\
{[1.50]}\end{array}$ & $\begin{array}{c}0.8 \\
{[0.17]}\end{array}$ & $\begin{array}{c}33.1 \\
{[3.66]}\end{array}$ & $\begin{array}{c}1.1 \\
{[0.14]}\end{array}$ & $\begin{array}{c}0.44 \\
{[0.06]}\end{array}$ \\
\hline $\mathrm{T}-5 / 60 / 30$ & $\begin{array}{c}2.4 \\
{[0.25]}\end{array}$ & $\begin{array}{c}0.90 \\
{[0.02]}\end{array}$ & $\begin{array}{c}11.7 \\
{[0.46]}\end{array}$ & $\begin{array}{c}5.2 \\
{[0.57]}\end{array}$ & $\begin{array}{c}11.9 \\
{[0.92]}\end{array}$ & $\begin{array}{c}0.7 \\
{[0.08]}\end{array}$ & $\begin{array}{c}28.8 \\
{[1.31]}\end{array}$ & $\begin{array}{c}1.2 \\
{[0.15]}\end{array}$ & $\begin{array}{c}0.46 \\
{[0.02]}\end{array}$ \\
\hline
\end{tabular}


Córdova et al., Caracterización física y mecánica de compuestos de Guazuma...

\begin{tabular}{|c|c|c|c|c|c|c|c|c|c|}
\hline T-5/60/20 & $\begin{array}{c}1.4 \\
{[0.26]}\end{array}$ & $\begin{array}{c}0.90 \\
{[0.02]}\end{array}$ & $\begin{array}{c}9.1 \\
{[1.70]}\end{array}$ & $\begin{array}{c}4.1 \\
{[0.29]}\end{array}$ & $\begin{array}{c}13.4 \\
{[0.86]}\end{array}$ & $\begin{array}{c}0.8 \\
{[0.09]}\end{array}$ & $\begin{array}{c}29.3 \\
{[1.31]}\end{array}$ & $\begin{array}{c}1.2 \\
{[0.17]}\end{array}$ & $\begin{array}{c}0.44 \\
{[0.03]}\end{array}$ \\
\hline T-5/60/10 & $\begin{array}{c}1.8 \\
{[0.38]}\end{array}$ & $\begin{array}{c}0.83 \\
{[0.04]}\end{array}$ & $\begin{array}{c}9.1 \\
{[3.26]}\end{array}$ & $\begin{array}{c}4.0 \\
{[0.38]}\end{array}$ & $\begin{array}{c}13.2 \\
{[1.25]}\end{array}$ & $\begin{array}{c}0.7 \\
{[0.05]}\end{array}$ & $\begin{array}{c}29.8 \\
{[2.52]}\end{array}$ & $\begin{array}{c}1.1 \\
{[0.19]}\end{array}$ & $\begin{array}{c}0.44 \\
{[0.02]}\end{array}$ \\
\hline T-5/80/30 & $\begin{array}{c}2.0 \\
{[0.22]}\end{array}$ & $\begin{array}{c}0.93 \\
{[0.01]}\end{array}$ & $\begin{array}{c}10.6 \\
{[0.55]}\end{array}$ & $\begin{array}{c}4.9 \\
{[1.04]}\end{array}$ & $\begin{array}{c}11.2 \\
{[0.89]}\end{array}$ & $\begin{array}{c}0.6 \\
{[0.03]}\end{array}$ & $\begin{array}{c}25.6 \\
{[1.81]}\end{array}$ & $\begin{array}{c}1.0 \\
{[0.08]}\end{array}$ & $\begin{array}{c}0.45 \\
{[0.04]}\end{array}$ \\
\hline T-5/80/20 & $\begin{array}{c}1.3 \\
{[0.14]}\end{array}$ & $\begin{array}{c}0.90 \\
{[0.01]}\end{array}$ & $\begin{array}{c}8.4 \\
{[1.45]}\end{array}$ & $\begin{array}{c}4.2 \\
{[0.55]}\end{array}$ & $\begin{array}{c}13.7 \\
{[0.64]}\end{array}$ & $\begin{array}{c}0.7 \\
{[0.06]}\end{array}$ & $\begin{array}{c}27.4 \\
{[1.65]}\end{array}$ & $\begin{array}{c}1.0 \\
{[0.07]}\end{array}$ & $\begin{array}{c}0.45 \\
{[0.01]}\end{array}$ \\
\hline T-5/80/10 & $\begin{array}{c}2.1 \\
{[0.41]}\end{array}$ & $\begin{array}{c}0.86 \\
{[0.01]}\end{array}$ & $\begin{array}{c}6.0 \\
{[2.13]}\end{array}$ & $\begin{array}{c}3.3 \\
{[0.62]}\end{array}$ & $\begin{array}{c}12.9 \\
{[0.71]}\end{array}$ & $\begin{array}{c}0.9 \\
{[0.11]}\end{array}$ & $\begin{array}{c}27.3 \\
{[3.20]}\end{array}$ & $\begin{array}{c}0.9 \\
{[0.15]}\end{array}$ & $\begin{array}{c}0.47 \\
{[0.04]}\end{array}$ \\
\hline T-6/40/30 & $\begin{array}{c}2.0 \\
{[0.16]}\end{array}$ & $\begin{array}{c}0.90 \\
{[0.01]}\end{array}$ & $\begin{array}{c}11.6 \\
{[0.64]}\end{array}$ & $\begin{array}{c}5.2 \\
{[0.46]}\end{array}$ & $\begin{array}{c}12.3 \\
{[0.95]}\end{array}$ & $\begin{array}{c}0.8 \\
{[0.10]}\end{array}$ & $\begin{array}{c}29.3 \\
{[5.07]}\end{array}$ & $\begin{array}{c}1.2 \\
{[0.21]}\end{array}$ & $\begin{array}{c}0.48 \\
{[0.04]}\end{array}$ \\
\hline T-6/40/20 & $\begin{array}{c}1.6 \\
{[0.17]}\end{array}$ & $\begin{array}{c}0.88 \\
{[0.01]}\end{array}$ & $\begin{array}{c}6.4 \\
{[1.22]}\end{array}$ & $\begin{array}{c}3.7 \\
{[0.63]}\end{array}$ & $\begin{array}{c}16.8 \\
{[0.65]}\end{array}$ & $\begin{array}{c}0.8 \\
{[0.06]}\end{array}$ & $\begin{array}{c}27.8 \\
{[1.50]}\end{array}$ & $\begin{array}{c}1.1 \\
0.11]\end{array}$ & $\begin{array}{c}0.42 \\
{[0.03]}\end{array}$ \\
\hline T-6/40/10 & $\begin{array}{c}1.4 \\
{[0.11]}\end{array}$ & $\begin{array}{c}0.82 \\
{[0.01]}\end{array}$ & $\begin{array}{c}5.1 \\
{[1.54]}\end{array}$ & $\begin{array}{c}4.0 \\
{[1.14]}\end{array}$ & $\begin{array}{c}18.8 \\
{[0.84]}\end{array}$ & $\begin{array}{c}0.8 \\
{[0.09]}\end{array}$ & $\begin{array}{c}31.4 \\
{[3.07]}\end{array}$ & $\begin{array}{c}1.0 \\
{[0.11]}\end{array}$ & $\begin{array}{c}0.52 \\
{[0.02]}\end{array}$ \\
\hline T-6/60/30 & $\begin{array}{c}2.1 \\
{[0.11]}\end{array}$ & $\begin{array}{c}0.90 \\
{[0.01]}\end{array}$ & $\begin{array}{c}10.8 \\
{[0.96]}\end{array}$ & $\begin{array}{c}5.2 \\
{[0.71]}\end{array}$ & $\begin{array}{c}12.7 \\
{[0.79]}\end{array}$ & $\begin{array}{c}0.7 \\
{[0.11]}\end{array}$ & $\begin{array}{c}25.5 \\
{[0.47]}\end{array}$ & $\begin{array}{c}1.0 \\
{[0.09]}\end{array}$ & $\begin{array}{c}0.46 \\
{[0.02]}\end{array}$ \\
\hline T-6/60/20 & $\begin{array}{c}1.7 \\
{[0.19]}\end{array}$ & $\begin{array}{c}0.86 \\
{[0.02]}\end{array}$ & $\begin{array}{c}6.0 \\
{[0.39]}\end{array}$ & $\begin{array}{c}3.6 \\
{[0.63]}\end{array}$ & $\begin{array}{c}14.4 \\
{[1.37]}\end{array}$ & $\begin{array}{c}0.7 \\
{[0.09]}\end{array}$ & $\begin{array}{c}29.0 \\
{[1.92]}\end{array}$ & $\begin{array}{c}1.2 \\
{[0.07]}\end{array}$ & $\begin{array}{c}0.48 \\
{[0.03]}\end{array}$ \\
\hline $\mathrm{T}-6 / 60 / 10$ & $\begin{array}{c}1.6 \\
{[0.39]}\end{array}$ & $\begin{array}{c}0.84 \\
{[0.02]}\end{array}$ & $\begin{array}{c}6.6 \\
{[2.41]}\end{array}$ & $\begin{array}{c}3.6 \\
{[1.74]}\end{array}$ & $\begin{array}{c}15.0 \\
{[1.77]}\end{array}$ & $\begin{array}{c}0.8 \\
{[0.07]}\end{array}$ & $\begin{array}{c}33.6 \\
{[1.12]}\end{array}$ & $\begin{array}{c}1.1 \\
{[0.03]}\end{array}$ & $\begin{array}{c}0.46 \\
{[0.03]}\end{array}$ \\
\hline T-6/80/30 & $\begin{array}{c}2.7 \\
{[0.45]}\end{array}$ & $\begin{array}{c}0.90 \\
{[0.03]}\end{array}$ & $\begin{array}{c}9.9 \\
{[1.30]}\end{array}$ & $\begin{array}{c}5.0 \\
{[1.25]}\end{array}$ & $\begin{array}{c}10.7 \\
{[0.25]}\end{array}$ & $\begin{array}{c}0.7 \\
{[0.10]}\end{array}$ & $\begin{array}{c}26.9 \\
{[0.98]}\end{array}$ & $\begin{array}{c}1.1 \\
{[0.10]}\end{array}$ & $\begin{array}{c}0.44 \\
{[0.02]}\end{array}$ \\
\hline T-6/80/20 & $\begin{array}{c}1.8 \\
{[0.28]}\end{array}$ & $\begin{array}{c}0.90 \\
{[0.01]}\end{array}$ & $\begin{array}{c}6.8 \\
{[1.13]}\end{array}$ & $\begin{array}{c}4.3 \\
{[0.97]}\end{array}$ & $\begin{array}{c}13.2 \\
{[0.91]}\end{array}$ & $\begin{array}{c}0.7 \\
{[0.06]}\end{array}$ & $\begin{array}{c}27.2 \\
{[3.19]}\end{array}$ & $\begin{array}{c}1.1 \\
{[0.19]}\end{array}$ & $\begin{array}{c}0.47 \\
{[0.01]}\end{array}$ \\
\hline T-6/80/10 & $\begin{array}{c}1.2 \\
{[0.19]}\end{array}$ & $\begin{array}{c}0.84 \\
{[0.01]}\end{array}$ & $\begin{array}{c}7.8 \\
{[2.12]}\end{array}$ & $\begin{array}{c}4.7 \\
{[0.77]}\end{array}$ & $\begin{array}{c}14.2 \\
{[1.92]}\end{array}$ & $\begin{array}{c}0.9 \\
{[0.04]}\end{array}$ & $\begin{array}{c}31.0 \\
{[1.74]}\end{array}$ & $\begin{array}{c}1.1 \\
{[0.06]}\end{array}$ & $\begin{array}{c}0.51 \\
{[0.04]}\end{array}$ \\
\hline
\end{tabular}


Cuadro 3. Dimensiones de elementos anatómicos de interés en fibras de Guazuma crinita Mart. de 4, 5 y 6 años de edad.

\begin{tabular}{lccc}
\hline Característica / Edad (años) & $\mathbf{4}$ & $\mathbf{5}$ & $\mathbf{6}$ \\
\hline Longitud de fibra $(\mu \mathrm{m})$ & 1399 & 1554 & 1100 \\
Diámetro total de fibra $(\mu \mathrm{m})$ & {$[496.3]$} & {$[294.7]$} & {$[141.6]$} \\
& 26.7 & 27.9 & 26.3 \\
Espesor de pared de fibra $(\mu \mathrm{m})$ & {$[6.4]$} & {$[5.49]$} & {$[6.54]$} \\
Diámetro lumen de fibra $(\mu \mathrm{m})$ & 2.1 & 2.1 & 2.1 \\
& {$[0.3]$} & {$[0.36]$} & {$[0.3]$} \\
Coeficiente de esbeltez & 22.4 & 23.7 & 22.0 \\
& {$[6.4]$} & {$[5.3]$} & {$[6.58]$} \\
& 53.4 & 57.4 & 44.1 \\
& {$[19.2]$} & {$[14.76]$} & {$[10.4]$} \\
\hline
\end{tabular}

\section{Caracterización anatómica}

El análisis de las fibras se hizo por su posible influencia en las propiedades físicas y mecánicas del material compuesto.

La longitud promedio de fibra fue mayor para la edad de 5 años (1 $554 \mu \mathrm{m}$ ), seguido de la de 4 años (1 $399 \mu \mathrm{m}$ ) y la de 6 años $(1100 \mu \mathrm{m})$. De acuerdo con IAWA (1989), dichas dimensiones son catalogadas como fibras de longitud mediana (900-1 $600 \mu \mathrm{m})$; por lo tanto, a todas se les consideró así. El diámetro de fibra promedio fue de 28,27 y $26 \mu \mathrm{m}$, para las edades 5,4 y 6 años sin que se confirmara una diferencia significativa.

Según Ibama (1991), el diámetro de fibra se clasifica como mediano. El espesor de pared promedio para las tres edades $(2 \mu \mathrm{m})$ se define como muy delgada (IAWA, 1989). El coeficiente de esbeltez (conocido también como la relación largo/ancho) registró su valor más alto para los 5 años de edad (57), seguido de la de 4 años (53) y la de 6 años (44). Los resultados se muestran en el Cuadro 3. 


\section{Caracterización química}

Los contenidos de extractivos, holocelulosa, lignina y cenizas resultaron muy semejantes para las tres edades evaluadas. El contenido de extractivos y de cenizas fueron numéricamente inferiores a los registrados por Oluwadare y Asagbara (2008), quienes en un estudio de la composición química de Sterculia setigera Delile, especie de misma familia que Guazuma crinita (Cuadro 4). Los resultados de contenido de lignina guardaron semejanza numérica con los valores hallados por Oluwadare y Asagbara (2008). El contenido de holocelulosa fue superior al consignado por Pettersen (1984) para Guazuma tomentosa Kunth.

Cuadro 4. Caracterización química de madera de Guazuma crinita Mart. de 4, 5 y 6 años de edad.

\begin{tabular}{lccc}
\hline Característica / Edad (años) & $\mathbf{4}$ & $\mathbf{5}$ & $\mathbf{6}$ \\
\hline Extractivos (\%) & 3.08 & 1.98 & 2.11 \\
& {$[1.01]$} & {$[0.60]$} & {$[0.21]$} \\
Holocelulosa (\%) & 72.01 & 75.43 & 72.36 \\
& {$[3.40]$} & {$[6.27]$} & {$[0.36]$} \\
Lignina (\%) & 22.75 & 21.81 & 24.27 \\
& {$[0.59]$} & {$[1.57]$} & {$[1.16]$} \\
Cenizas (\%) & 1.21 & 1.22 & 1.11 \\
& {$[0.18]$} & {$[0.04]$} & {$[0.14]$} \\
\hline
\end{tabular}

\section{Propiedades físicas}

\section{Contenido de Humedad}

En la Figura 1 se presentan los valores promedio de humedad final para todas las muestras del material compuesto PP-Bolaina blanca, los cuales oscilan entre 2.2 a $0.9 \%$ para el año 4 , de 2.7 a $1.3 \%$ para el año 5 y de 2.1 a $1.2 \%$ para el año 6 . Los contenidos de humedad guardan similitud numérica en las tres edades consideradas, a pesar de que estadísticamente resultó como variable influyente. La composición química de las fibras de $G$. crinata casi no registró diferencias en sus tres edades. 


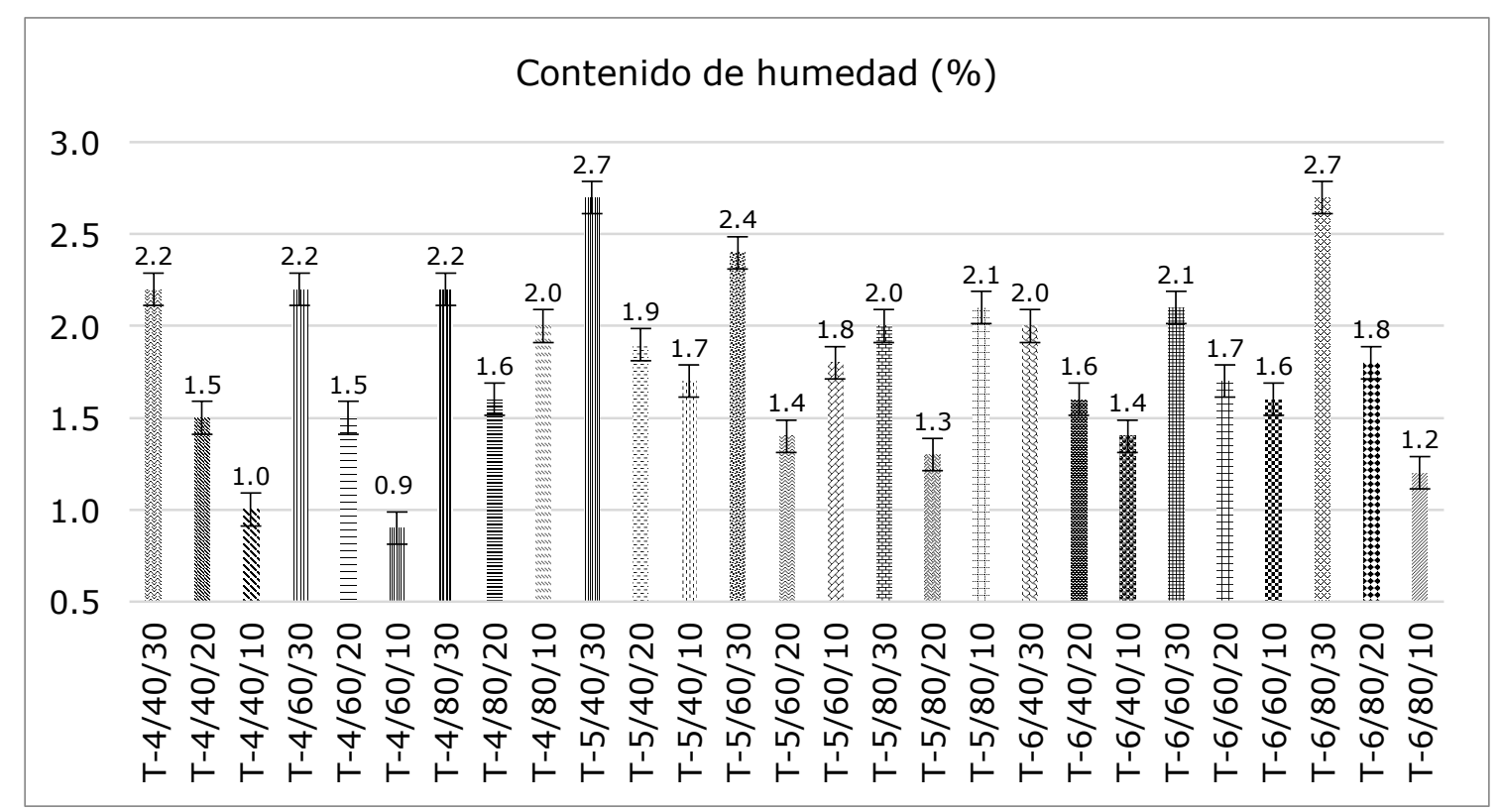

Figura 1. Contenido de humedad de los materiales compuestos PP-Bolaina blanca.

La proporción de partículas en el material compuesto PP-Bolaina blanca y la humedad final evidenciaron una relación proporcional, la cual obedece a la naturaleza higroscópica inherente de la madera. Los carbohidratos que conforman la pared celular de las fibras vegetales, tales como celulosa y hemicelulosas, incluyen grupos hidroxilo $(\mathrm{OH})$, los que son bastante afines al agua (Caulfield et al., 2005; Bouafif et al., 2009). Asimismo, el contenido promedio de holocelulosa (celulosa y hemicelulosas) en las fibras de G.crinita para las tres edades en estudio está por encima de $70 \%$, lo que confirma una fuerte afinidad entre las fibras y la humedad circundante. En materiales compuestos plástico-madera, los componentes lignocelulósicos son responsables de la ganancia de humedad; las matrices por lo general poseen carácter hidrófobo (Klyosov, 2007; Caicedo et al., 2015).

Cárdenas (2012) hace referencia a un intervalo máximo de aceptabilidad para el contenido de humedad en materiales compuestos de $2 \%$. El mismo autor registró valores de 0.27 a $0.31 \%$ para materiales compuestos elaborados por el método de inyección. 
El análisis estadístico indicó que las variables edad y proporción de mezcla son altamente significativas ( $p \leq 0.0041)$. La interacción doble edad*tamaño de partícula y la interacción triple afectan de igual manera a los valores de humedad ( $p \leq 0.0040)$.

\section{Densidad aparente}

La Figura 2 presenta los valores promedio de densidad aparente para todas las muestras del material compuesto PP-Bolaina blanca. Los valores oscilan entre 0.91 a $0.76 \mathrm{~g} . \mathrm{cm}^{-3}$ para el año 4 , de 0.93 a $0.83 \mathrm{~g} \cdot \mathrm{cm}^{-3}$ para el año 5 y de 0.90 a $0.82 \mathrm{~g} . \mathrm{cm}^{-3}$ para el año 6 . La edad 5 años registra los valores más altos en densidad aparente; no obstante, la caracterización anatómica y química practicadas a las fibras de $G$. crinita resultaron con poca diferencia para las tres edades en estudio.



Figura 2. Densidad aparente de los materiales compuestos PP-Bolaina blanca. 
El análisis estadístico sugirió que las variables edad, tamaño de partícula y proporción de mezcla ejercen influencia altamente significativa $(p \leq 0.0015)$; sin embargo, las interacciones dobles y la interacción múltiple no actuaron de esa forma sobre la densidad $(p \geq 0.0405)$.

Respecto al tamaño de partículas, los tratamientos que incluyen las partículas más pequeñas registraron los valores de densidad más altos, dada la mayor facilidad de encapsulamiento del material (Fabiyi, 2007; Klyosov, 2007; Cárdenas, 2012). Asimismo, se aprecia un ligero incremento en la densidad, cuando aumenta la proporción de partículas en el material compuesto. A pesar de que la madera de bolaina blanca presenta una densidad baja, el incremento de la misma favorece la densidad del material compuesto.

Moya et al. (2012) calcularon valores de entre 0.98 y $1.04 \mathrm{~g} \cdot \mathrm{cm}^{-3}$ para materiales compuestos reforzados con aserrín de pino. Cárdenas (2012) determinó densidades de 1.06 a $1.11 \mathrm{~g} . \mathrm{cm}^{-3}$ para materiales compuestos de polipropileno y madera de pino elaborados por el método de inyección; Lázaro et al. (2016a) dieron a conocer resultados similares, mediante el método combinado de extrusión y compresión. A su vez, Soatthiyanon (2010) consignó densidades entre 1.01 y $1.14 \mathrm{~g} \mathrm{.}^{-3}$ para diferentes tipos de materiales compuestos. Los números de densidad aparente generados en el presente estudio fueron inferiores, con respecto a los de la literatura citada.

\section{Absorción e hinchamiento}

La Figura 3 exhibe los valores promedio de absorción e hinchamiento para el material compuesto PP-Bolaina blanca durante dos meses de inmersión en agua. 


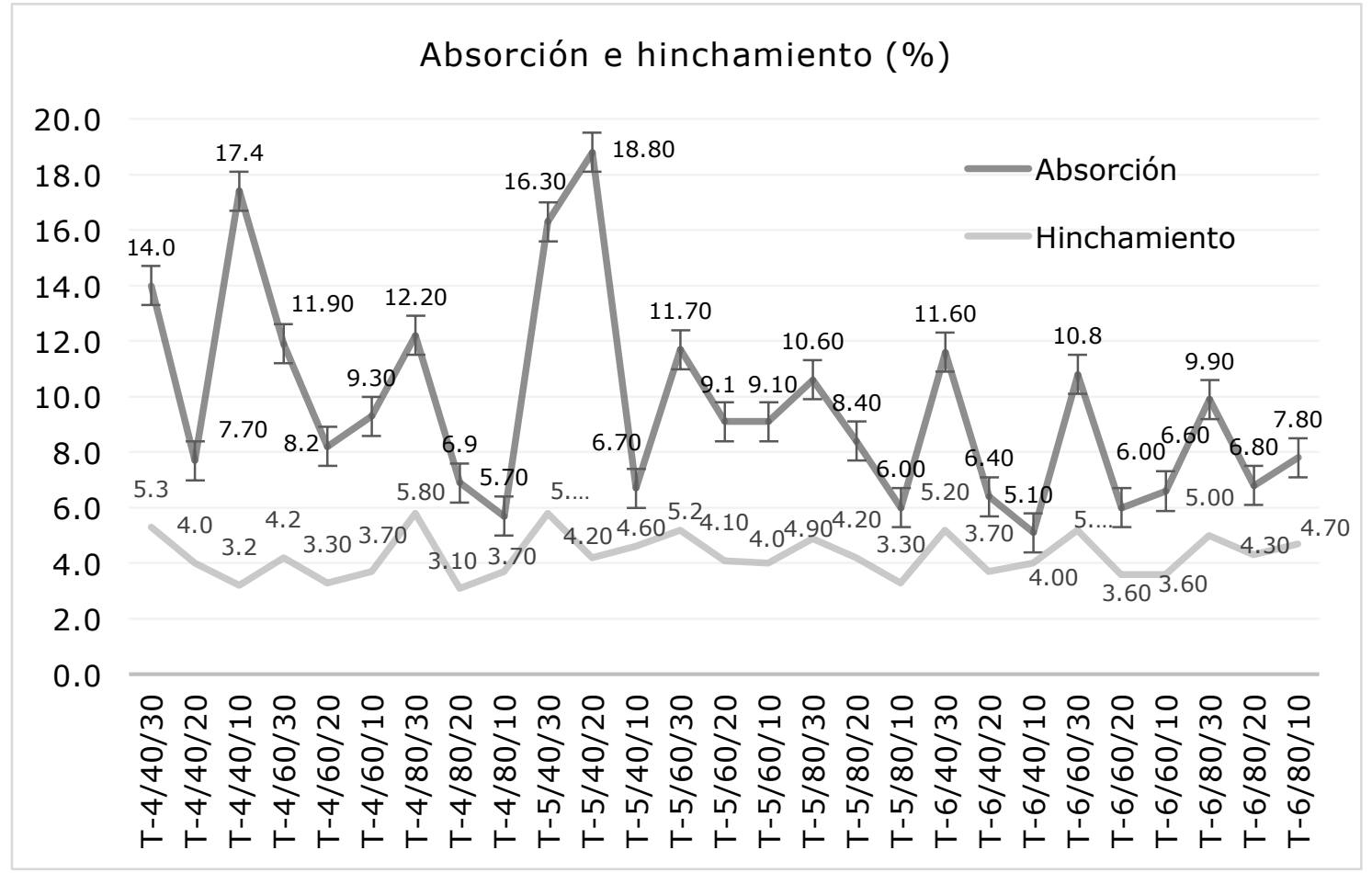

Figura 3. Valores promedio de absorción e hinchamiento en materiales compuestos PP-Bolaina blanca.

Los valores promedio de absorción varían de 17.4 a $5.7 \%$ para el año 4, de 18.8 a $6.0 \%$ para el año 5 y de 11.6 a $5.1 \%$ para el año 6 . A pesar que la variable edad presentó diferencia altamente significativa $(p \leq 0.0001)$, la composición química de las fibras de G.crinita para las tres edades fue muy similar, por lo que no influyó en estos resultados.

Se aprecia una ligera disminución de los valores de absorción al emplear partículas de menor tamaño, lo cual concuerda con Fabiyi (2007) y con Fuentes-Talavera et al. (2014). Cuando la interface en el material compuesto es homogénea y compacta, los elementos fibrosos están embebidos dentro de la matriz e imposibilitados de absorber humedad del exterior. Las partículas grandes son difíciles de embeber por la matriz y dejan regiones expuestas por donde absorberán humedad (Simonsen y Rials, 1996; Caulfield et al., 2005). Asimismo, se reconoce una relación directa entre los valores de absorción y la proporción de partículas. Klyosov (2007) señala que la mayoría de plásticos utilizados en materiales compuestos, prácticamente, no absorben agua; por consiguiente, la incorporación de partículas celulósicas es la responsable de incrementar significativamente la absorción de agua. 
Soattiyanon (2010) registró valores de absorción entre 8 y 9 \% para diferentes tipos de materiales compuestos durante periodos de inmersión superiores a 6 meses, en materiales procesados por inyección; proceso que asegura un mejor recubrimiento de la fibra y por ende una mayor resistencia a la absorción. A su vez, Lázaro et al. (2016a) calcularon valores de absorción de 14 a $15 \%$ en materiales compuestos de polipropileno y bambú, resultados similares a los de la presente investigación.

El análisis estadístico indicó una influencia altamente significativa $(p \leq 0.0001)$ para las tres variables; del mismo modo, las interacciones dobles edad*tamaño de partícula y edad*proporción de mezcla, así como la interacción triple, afectaron los valores de absorción ( $p \geq 0.0007)$.

Los valores promedio de hinchamiento variaron entre 5.8 y $3.1 \%$ para el año 4 , de 5.8 a $3.3 \%$ para el año 5 y de 5.2 a $3.6 \%$ para el año 6 . Las edades de 5 y 6 años tuvieron el mayor aumento de hinchamiento para las dos primeras horas de inmersión en agua. Por lo general, los tratamientos con partículas más grandes alcanzaron los valores más altos de hinchamiento, dado que no se encapsula totalmente la fibra, tendencia consignada en diferentes estudios (Okubo et al., 2004; Mattos et al., 2014). Se define una relación directa entre el hinchamiento y la proporción de partículas, tal como sucede en la absorción. Esto se debe a la naturaleza hidrofílica de las partículas de madera, en especial por la presencia de grupos hidroxilo $(\mathrm{OH})$ en la celulosa y hemicelulosas, componentes mayoritarios en la madera (Caulfield et al., 2005; Bouafif et al., 2009).

Cárdenas (2012) declaró incrementos en el hinchamiento cercanos a $10 \%$ en materiales compuestos reforzados con madera de pino (50 por ciento del peso total), para periodos de inmersión superiores a cinco meses. En otra investigación, Gonzáles et al. (2018) registraron cifras de $2.6 \%$ en materiales compuestos con bambú (30\% del peso total), para las primeras 24 h de inmersión y en materiales elaborados por extrusión y compresión. Los valores de hinchamiento obtenidos en el presente estudio superaron a los indicados por Gonzáles et al. (2018), pero fueron inferiores a los de Cárdenas (2012), quien experimentó con periodos de inmersión más prolongados y materiales compuestos elaborados por inyección. 
El análisis estadístico indicó que la variable proporción de mezcla presentó influencia altamente significativa $(p=0.0001)$ sobre los valores de hinchamiento.

\section{Propiedades mecánicas}

\section{Módulo de ruptura (MOR)}

La Figura 4 reúne los valores promedio de módulo de ruptura (MOR) en flexión estática y tensión para todas las muestras del material compuesto PP-Bolaina blanca. Los valores promedio de MOR en flexión variaron entre 33.7 y $26.2 \mathrm{MPa}$ para el año 4, de 33.1 a $25.6 \mathrm{MPa}$ para el año 5 y de 33.6 a $25.5 \mathrm{MPa}$ para el año 6.

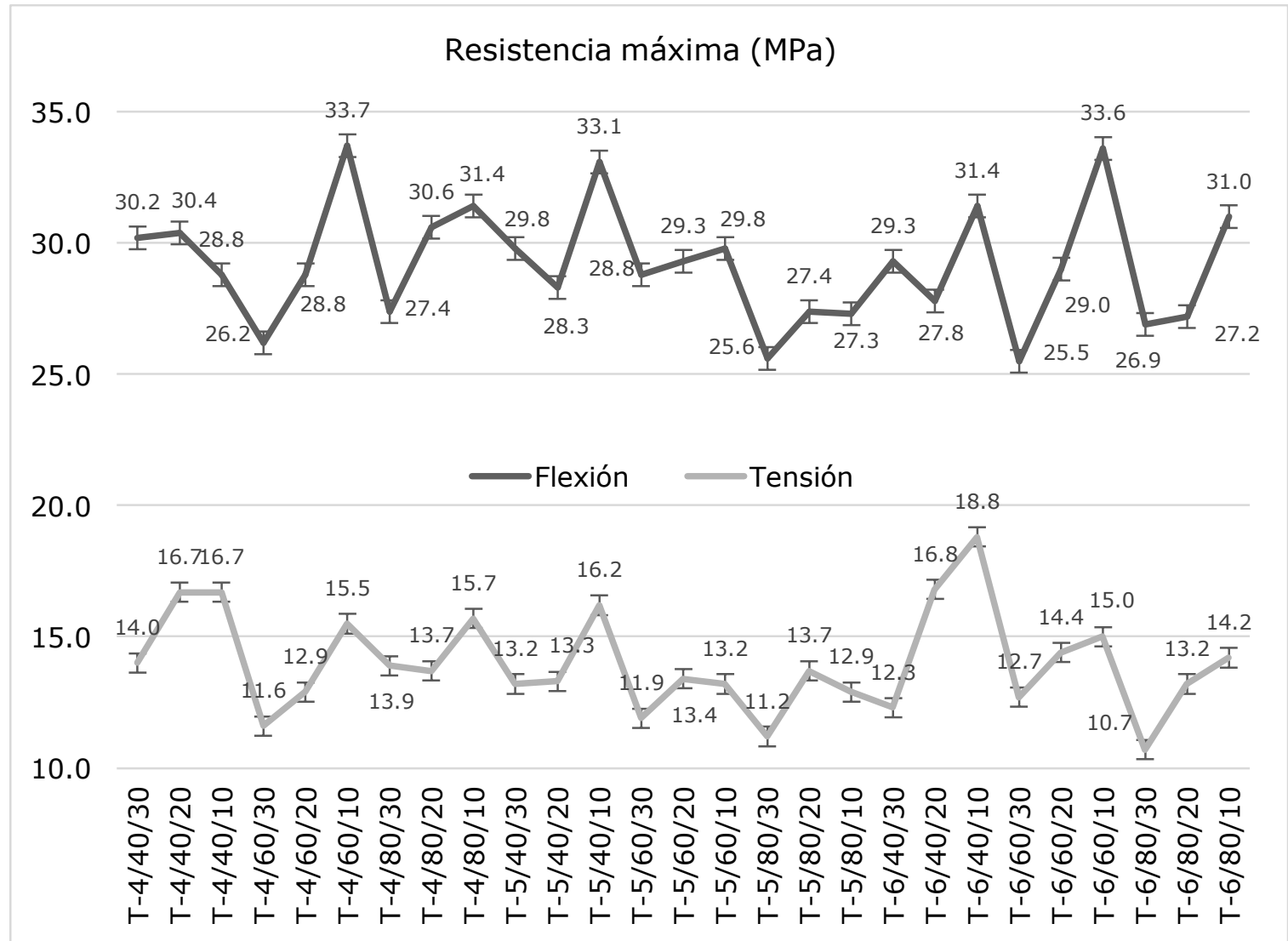

Figura 4. Módulo de ruptura (MOR) en flexión estática y tensión paralela de los materiales compuestos PP-Bolaina blanca. 
Se aprecia una relación inversa entre la proporción de partículas y los valores de MOR en flexión, en la que los tratamientos con menos proporción de partículas alcanzaron valores altos de MOR. De acuerdo a Klyosov (2007), conforme aumenta la proporción de fibras lignocelulósicas en material compuesto, el MOR empieza a decrecer.

Los valores de MOR en flexión obtenidos en el estudio que aquí se documenta se asemejan a los referidos por Cárdenas (2012) y Klyosov (2007) (31-34 MPa y 21$26 \mathrm{MPa}$, respectivamente). Otros investigadores, como Idrus et al. (2011) y Ravi et al. (2014) coinciden en afirmar lo contrario: una mayor cantidad de fibras en el material compuesto mejora los valores de MOR en flexión. Una explicación para este comportamiento es la deficiente interacción refuerzo/matriz en el material compuesto, debido al método de fabricación, así como a las características anatómicas (longitud y espesor de pared) de las fibras de G. crinita. Es preciso señalar que la responsabilidad de conferir resistencia mecánica al material compuesto recae en las fibras. El análisis estadístico señaló para la variable proporción de mezcla una influencia altamente significativa $(p=0.0001)$ sobre los valores de MOR en flexión.

Los valores promedio de MOR en tensión se registraron en un intervalo de 16.7 y 11.6 MPa para el año 4, de 16.2 a 11.2 MPa para el año 5 y de 18.8 a 10.7 MPa para el año 6. Los de MOR en tensión tuvieron similitud numérica en las tres edades estudiadas, a pesar de que estadísticamente la edad es una variable influyente. El coeficiente de esbeltez de la fibra de G. crinita casi no tuvo diferencias en sus tres edades.

Respecto al tamaño de las partículas, se aprecia un incremento moderado de los valores del MOR en tensión, si se utilizan piezas más grandes. Stark y Berger (1997) argumentaron que dicha característica es mayor cuando aumenta el tamaño de

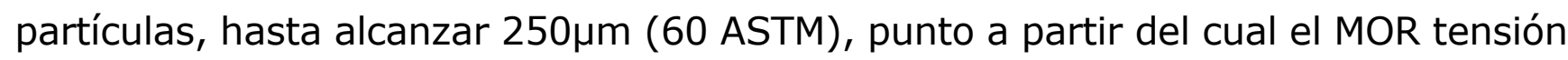
empieza a decrecer. Sin embargo, autores como Bledzki et al. (2005) y Nourbakhsh et al., (2010) sostienen que la resistencia a la tensión en materiales compuestos PP-madera crece al disminuir el tamaño de las partículas, y atribuyeron este comportamiento a una mejora en la adhesión interfacial entre las partículas de madera y la matriz. 
Se verificó un incremento significativo en los valores del MOR en tensión cuando se reduce la proporción de partículas de madera en el material compuesto $(p=0.0001)$. Este fenómeno ha sido señalado por otros investigadores (Klyosov, 2007; Ravi et al., 2014), que coinciden al afirmar que altas concentraciones de partículas de madera reducen el MOR del material compuesto.

Caulfield et al. (2005) registraron valores de MOR en tensión de $44.9 \mathrm{MPa}$ para materiales compuestos de polipropileno y fibras de álamo (30 por ciento del peso total). Stark y Rowlands (2003) consignaron valores de MOR de 29.4 y $37 \mathrm{MPa}$ para materiales compuestos con aserrín en 40 y $20 \%$ del peso total, respectivamente. Cárdenas (2012) registró valores de MOR entre 19 y $25 \mathrm{MPa}$ para materiales compuestos polipropileno y madera de pino elaborados por el método de inyección. Para las variables edad, tamaño de partículas y proporción de partículas, el análisis estadístico indicó una influencia altamente significativa $(p=0.0001)$; del mismo modo, las interacciones dobles edad*tamaño de partícula y edad*proporción de mezcla, así como la interacción triple incidieron sobre los valores de MOR en tensión $(p \leq 0.0093)$.

\section{Módulo de elasticidad (MOE)}

La Figura 5 exhibe los valores promedio de módulo de elasticidad (MOE) en flexión estática y tensión para todas las muestras del material compuesto PP-Bolaina blanca. Los valores promedio de MOE en flexión estática fluctuaron entre 1.3 y 0.9 GPa para el año 4, de 1.2 a 0.9 GPa para el año 5 y de 1.2 a $1.0 \mathrm{GPa}$ para el año 6. 


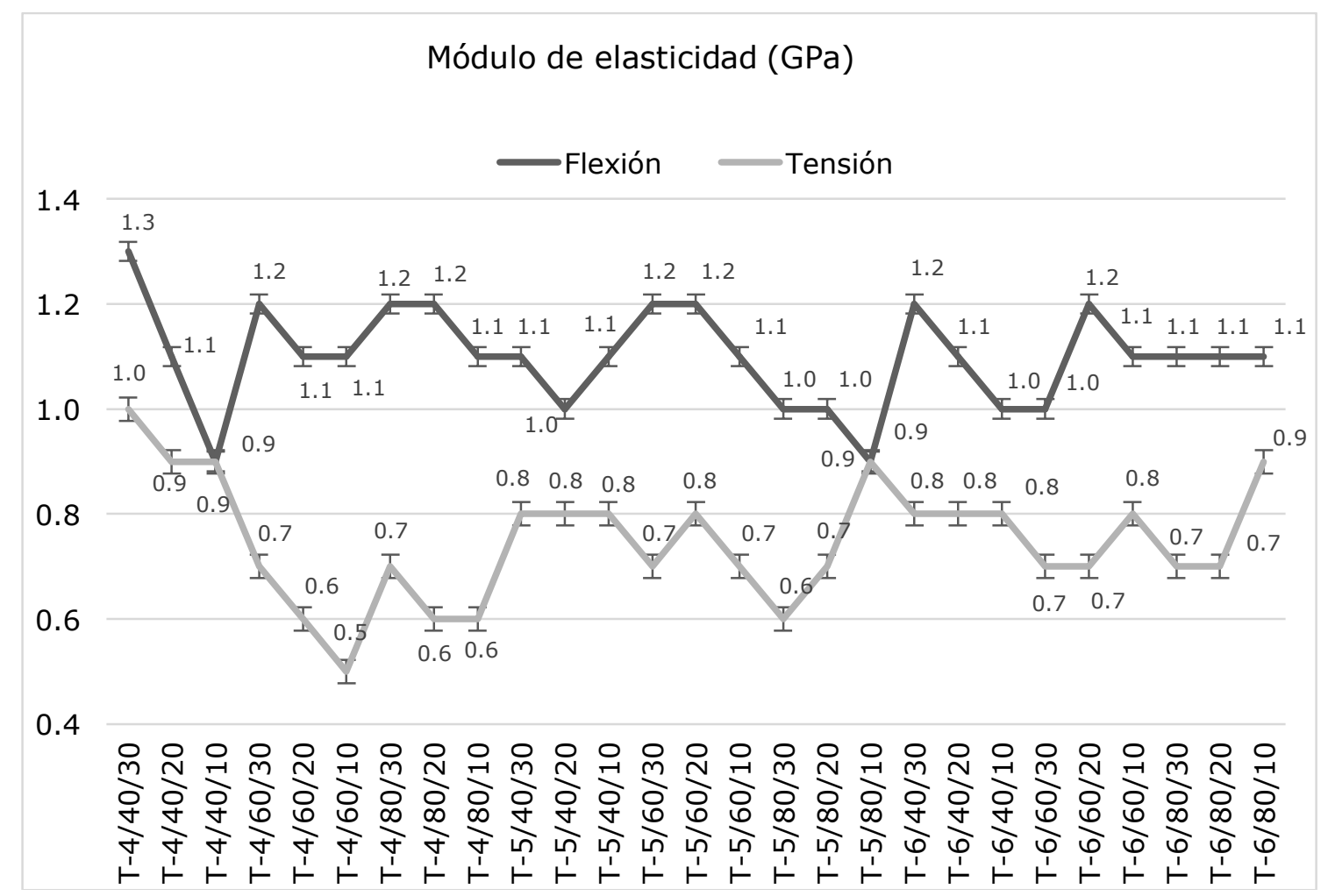

Figura 5. Módulo de elasticidad (MOE) en flexión estática y tensión de los materiales compuestos PP-Bolaina blanca.

Los tratamientos con mayor proporción de partículas obtuvieron valores más altos de MOE en flexión. Las fibras de madera, generalmente, presentan buen comportamiento a la flexión, por lo cual los materiales compuestos con mayor cantidad de fibras demandan más esfuerzo para lograr su deformación (Caulfield et al., 2005; Idrus et al., 2011). Sin embargo, reforzar con más partículas el material compuesto no produce, necesariamente, mejoras en el MOE en flexión. Ravi et al. (2014) señalan que los espacios vacíos, la baja interacción entre fibras y una deficiente dispersión de las mismas en la matriz, influyen en forma negativa en las propiedades mecánicas del material compuesto.

Para la variable proporción de mezcla, con el el análisis estadístico se obtuvo una influencia altamente significativa $(p=0.0017)$ en los valores de MOE en flexión estática. Los valores promedio de MOE en tensión variaron de 1.0 a $0.5 \mathrm{GPa}$ para el año 4, de 0.9 a $0.6 \mathrm{GPa}$ para el año 5 y de 0.9 a $0.7 \mathrm{GPa}$ para el año 6 .

Se observó un leve incremento en los valores del MOE en tensión cuando se emplearon partículas de G.crinita más grandes, tal como sucedió con el módulo de 
ruptura. Los valores más altos de MOE en tensión correspondieron a los tratamientos con partículas de mayor tamaño y resultaron inferiores a los citados por Caulfield et al. (2005); Lisperguer et al. (2013) y Cárdenas (2012).

La baja adhesión interfacial entre las partículas de G.crinita y la matriz de polipropileno posiblemente generaron zonas de alta heterogeneidad en el interior del material compuesto, lo que mermó su resistencia a la deformación (Essabir et al., 2015). Asimismo, las características anatómicas de sus fibras en las tres edades bajo estudio registraron valores bajos, con longitudes de fibras de tamaño mediano y espesores de pared muy delgadas, características poco deseadas para ensayos de tensión (García et al., 2003).

Para la variable tamaño de partícula, el análisis estadístico indicó una influencia altamente significativa $(p=0.0001)$; del mismo modo, las interacciones dobles edad*tamaño de partícula y edad*proporción de mezcla presentaron influencia altamente significativa sobre los valores de MOE en tensión ( $p \leq 0.0005)$.

\section{Resistencia al impacto}

La Figura 6 presenta los valores promedio de resistencia al impacto para todas las muestras del material compuesto PP-Bolaina blanca. Los valores oscilaron de 0.62 a $0.46 \mathrm{~J}$ para el año 4 , de 0.47 a $0.44 \mathrm{~J}$ para el año 5 y de 0.52 a $0.42 \mathrm{~J}$ para el año 6 . 




Figura 6. Resistencia al impacto de materiales compuestos polipropileno Bolaina blanca.

Los valores para la edad de 4 años fueron ligeramente más altos, a pesar de que el coeficiente de esbeltez de la fibra casi no tuvo diferencias en las tres edades.

Los tratamientos con partículas más grandes y pequeñas, respectivamente obtuvieron los valores de resistencia al impacto más elevados. Este comportamiento irregular puede explicarse por la deficiente interacción refuerzo/matriz en el material compuesto, debido al método de fabricación. La presencia de madera como refuerzo en la matriz PP genera zonas en las que se concentra el esfuerzo, lo que propicia el inicio de grietas y la potencial falla del material compuesto (Nourbakhsh et al., 2010). Stark y Berger (1997) observaron que conforme aumentaba el tamaño de partícula, se incrementaba también la resistencia al impacto para diferentes materiales compuestos. Sin embargo, esto no se ajusta a los resultados del presente estudio en las que la matriz es la principal responsable de absorber la energía producida por el impacto. Durowaye et al. (2014) señalan que los incrementos en la cantidad de partículas de madera reducen la habilidad de absorber energía por parte de la 
matriz, lo que disminuye la resistencia al impacto en el material compuesto, influencia también consignada por Yuan et al. (2008).

El análisis estadístico indicó que las variables edad y tamaño de partícula ejercieron influencia altamente significativa $(p \leq 0.0002)$; del mismo modo, las interacciones dobles y la interacción triple afectaron los valores de resistencia al impacto $(p \leq 0.0004)$.

\section{Conclusiones}

La edad de la madera no representa una influencia numéricamente significativa, salvo para la propiedad física de absorción y la propiedad mecánica de resistencia al impacto. La proporción de partículas en el material compuesto PP-Bolaina blanca presenta una relación directa con las propiedades físicas de contenido de humedad, densidad, hinchamiento y absorción, así como con la propiedad mecánica de MOE en flexión estática; mientras que la relación es inversa, respecto a los valores de MOR en tensión y flexión estática, además de impacto.

El tamaño de partículas en el material compuesto PP-Bolaina blanca describe una relación directa, en relación a los valores de MOR y MOE en tensión.

\section{Agradecimientos}

A Javier Gago Campusano, del Instituto Peruano de Energía Nuclear (IPEN) por el apoyo en la extrusión de los materiales.

\section{Conflicto de intereses}

Los autores declaran no tener conflicto de intereses. 


\section{Contribución por autor}

Alexei Rodolfo Córdova Contreras: procesamiento de materia prima, elaboración de materiales compuestos, evaluación de propiedades físicas y mecánicas, análisis de datos; Aldo Joao Cárdenas Oscanoa: asesoría de investigación, redacción del manuscrito; Héctor Enrique Gonzáles Mora: planificación y asesoría de la investigación.

\section{Referencias}

American Society for Testing and Materials (ASTM). 1998. Standard Test Method for Water Absorption of Plastic. ASTM D 570 - 98. West Conshohocken, PA, USA. 4 p.

American Society for Testing and Materials (ASTM). 1999. Standard Test Methods for Evaluating Properties of Wood Base Fiber and Particle Panels Materials ASTM D 1037 - 99. West Conshohocken, PA, USA. 30 p.

American Society for Testing and Materials (ASTM). 2003a. Standard Test Methods for Flexural Properties of Unreinforced and Reinforced Plastics and Electrical Insulating Materials. ASTM D 790 - 03. West Conshohocken, PA, USA. 11 p. American Society for Testing and Materials (ASTM). 2003b. Standard Test Method for Tensile Properties for Plastics. ASTM D 638 - 03. West Conshohocken, PA, USA. 15 p.

American Society for Testing and Materials (ASTM). 2004. Standard Test Method for Impact Resistance of Flat, Rigid Plastic Specimen by Means of a Striker Impacted by a Falling Weight (Gardner Impact). ASTM D 5420 - 04. West Conshohocken, PA, USA. 8 p.

Bledzki, A. K., M. Letman, A. Viksne and L. Rence. 2005. A comparison of compounding processes and wood type for wood fibre-PP composites. Composites: Part A (36): 789-797. Doi: 10.1016/j.compositesa.2004.10.029. 
Bouafif, H., A. Koubaa, P. Perré and A. Cloutier. 2009. Effects of fiber characteristics on the physical and mechanical properties of wood plastic composites. Composites. Part A. 40 (12): 1975-1981. Doi:

10.1016/j.compositesa.2009.06.003.

Caicedo, C., A. Vásquez, L. Crespo, H. De La Cruz y O. Ossa. 2015. Material compuesto de matriz Polipropileno (PP) y fibra de Cedro: influencia del compatibilizante PP-g-MA. Informador Técnico (Colombia). 79 (2): 118-126. Doi: 10.23850/22565035.156.

Cárdenas, A. 2012. Evaluación de la presencia como preservante antifúngico del borato de zinc y su efecto en las propiedades físicas y mecánicas de materiales compuestos de plástico y madera de pino. Tesis de Maestría en Ciencia de Productos Forestales. Departamento de Madera, Celulosa y Papel. Universidad de Guadalajara. Guadalajara, Jal., México. 145 p.

Caulfield, D., C. Clemons, R. Jacobson and R. Rowell. 2005. Wood thermoplastic composites. Chapter 13. Handbook of wood chemistry and wood composite. CRC Press LLC. Boca Raton, FL, USA. pp. 366-379.

Correa, C., C. Razzino and E. Hage. 2007. Role of maleated coupling agents on the interface adhesion of polypropylene - wood composites. Journal of Thermoplastic Composite Materials 20 (3): 323-339. Doi: 10.1177/0892705707078896.

Clemons, C. 2002. Wood plastic composites in the United States: the interfacing of two industries. Forest Products Journal 52 (6): 10-18.

Durowaye, S., G. Lawal and O. Olagbaju. 2014. Microstructure and mechanical properties of sisal particles reinforced polypropylene composite. International Journal of Composite Materials 4 (4): 190-195. Doi:

10.5923/j.cmaterials.20140404.03.

Essabir, H., M. El Achaby, E. Hilali, R. Bouhfid, and A. Qaiss. 2015. Morphological, structural, thermal and tensile properties of high-density polyethylene composites reinforced with treated argan nutshell particles. Journal of Bionic Engineering. 12 (1): 129 - 141. Doi: 10.1016/S1672-6529(14)60107-4. 
Fabiyi, J. 2007. Chemistry of wood plastic composites weathering. Tesis de doctorado. College of graduated studies. University of Idaho. Idaho, ID, USA. 227 p.

Fuentes-Talavera, F., J. A. Silva-Guzmán, F. Quintana-Uscamayta, J. Turrado-Saucedo, A. J. Cárdenas O., R. Rodríguez-Anda y J. R. Robledo-Ortiz. 2014. Comportamiento al intemperismo natural de compositos polipropileno madera. Revista Mexicana de Ciencias Forestales 6 (27): 102-113. Doi: 10.29298/rmcf.v6i27.284.

García, L., A. Guindeo, C. Peraza, y P. De Palacios. 2003. La madera y su anatomía. Coedición Fundación Conde del Valle de Salazar. Mundi-Prensa. AiTiM. Madrid, España. 327 p.

Guerra, W., M. Soudre y M. Chota. 2008. Tabla de volumen comercial de Bolaina Blanca (Guazuma crinita Martius) de las plantaciones experimentales de Alexander von Humboldt, Ucayali, Perú. Folia Amazónica 17(1-2): 47-58. Doi: 10.24841/fa.v17i1-2.266.

Gonzáles, H., A. Cárdenas, y A. Becerra. 2018. Materiales compuestos: Investigación sobre materiales compuestos de bambú (Guadua angustifolia) y plástico (polipropileno). Innovate. Ministerio de la Producción. Lima, Perú. 182 p.

Instituto Brasileiro Do Meio Ambiente E Dos Recursos Naturais Renovaveis (Ibama). 1991. Normas de procedimiento em estudios do anatomia do madeira: I. Angiospermae. Brasilia, Brasil. 19 p.

International Association of Wood Anatomist (IAWA). 1989. List of microscopic features for hardwood identification. IAWA Bulletin New series 10:219-332.

Idrus, M., S. Hamdan, M. Rahman and M. Islam. 2011. Treated tropical wood sawdust-polypropylene polymer composite: mechanical and morphological study. Journal of Biomaterials and Nanobiotechnology 2 (4): 435 - 444. Doi: 10.4236/jbnb.2011.24053.

Klyosov, A. 2007. Wood-plastic composites. John Wiley \& Sons. Hoboken, NJ, USA. 726 p. 
Lázaro L., K. C., H. E. Gonzáles M., A. J. Cárdenas O. y J. Gago C. $2016 a$. Evaluación de las propiedades físicas de material compuesto elaborado con bambú (Guadua angustifolia Kunth) y polipropileno. Revista Mexicana de Ciencias Forestales 7 (38): 79-94. Doi: 10.29298/rmcf.v7i38.6.

Lázaro L., K. C., H. E. Gonzáles M. y A. J. Cárdenas O. 2016b. Propiedades mecánicas del material compuesto elaborado con bambú (Guadua angustifolia Kunth) y polipropileno. Revista Mexicana de Ciencias Forestales 7 (38): 95-110. Doi: $10.29298 /$ rmcf.v7i38.7.

Lisperguer, J., X. Bustos, Y. Saravia, C. Escobar y H. Venegas. 2013. Efecto de las características de harina de madera en las propiedades físico mecánicas y térmicas de polipropileno reciclado. Maderas. Ciencia y tecnología 15 (3): 321-336. Doi: $10.4067 /$ S0718-221X2013005000025.

Mattos, B., A. Misso, P. De Cademartori, E. De Lima, W. Magalhaes and D. Gatto. 2014. Properties of polypropylene composites filled with a mixture of household waste of mate-tea and wood particles. Construction and Building Materials 61: 6068. Doi: 10.1016/j.conbuildmat.2014.02.022.

Moya, C., H. Poblete y L. Valenzuela. 2012. Propiedades físicas y químicas de compuestos de polietileno reciclado y harinas de corteza y madera de Pinus radiata fabricados mediante moldeo por inyección. Maderas: Ciencia y Tecnología 14 (1): 13-29. Doi: $10.4067 /$ S0718-221X2012000100002.

Nourbakhsh, A., A. Karegarfard, A. Ashori and A. Nourbakssh. 2010. Effects of particle size and coupling agent concentration on mechanical properties of particulate-filled polymer composites. Journal of Thermoplastic Composite Materials. 23: 169-174. Doi: 10.1177/0892705709340492.

Okubo, K., T. Fujii and Y. Yamamoto. 2004. Development of bamboo-based polymer composites and their mechanical properties. Composites: Part A: applied science and manufacturing 35 (3): 377-383.

Doi: 10.1016/j.compositesa.2003.09.017. 
Oluwadare, A. O. and E. O. Asagbara. 2008. Biodegradation of Sterculia setigera (Sterculiaceae) chips and its effects on wood basic chemical composition. International Journal of Botany 4 (4): 461-465. Doi: 10.3923/ijb.2008.461.465.

Pettersen, R. 1984. The Chemistry of Solid Wood. American Chemical Society. Chapter 2. 57-126. Doi: 10.1021/ba-1984-0207.ch002.

Ravi, N., R. Ranga, R. Raghava B., and K. Srinivas. 2014. Mechanical properties of vakka fiber reinforced polypropylene composites. In: Thansekhar, M., N. Balaji. International Journal of Innovative Research in Science, Engineering and Technology 3(3): $1162-1166$.

Satyanarayana, K., G. Arizaga and F. Wypych. 2009. Biodegradable composites based on lignocellulosic fiber-An overview. Progress in Polymer Science 34 (9): 982-1021. Doi: 10.1016/j.progpolymsci.2008.12.002.

Simonsen, J. and T. Rials. 1996. Morphology and properties of wood-fiber reinforced blends of recycled polystyrene and polyethylene. Journal of Thermoplastic Composites Materials 9:292-302.

Doi: $10.1177 / 089270579600900306$.

Stark, N. and M. Berger. 1997. Effect of particle size on properties of wood-flour reinforced polypropylene composites. Proceedings of the Fourth International Conference on Woodfiber-Plastic Composites. Madison, WI, USA. pp. 134-143.

Stark, N. and R. Rowlands. 2003. Effects of wood fiber characteristics on mechanical properties of wood/polypropylene composites. Wood and Fiber Science 35(2): 167--174.

Soatthiyanon, N. 2010. Natural fiber reinforced polyolefin matrix composites. Materials Science and Engineering. The University of New South Wales. Sydney, Australia. 177 p.

Technical Association for the Pulp and Paper Industries (TAPPI). 1993. Ash wood, Pulp, paper and paperboard: combustion at $525^{\circ} \mathrm{C}$. TAPPI Test Method T 211 om93. Pechtree Corners, GA, USA. 4 p. 
Technical Association for the Pulp and Paper Industries (TAPPI). 1997a.

Preparation of wood for chemical analysis. TAPPI Test Method T 264 cm-97.

Pechtree Corners, GA, USA. 3 p.

Technical Association for the Pulp and Paper Industries (TAPPI). 1997b. Solvent extractives of wood and pulp. TAPPI Test Method T $204 \mathrm{~cm}-97$. Pechtree Corners, GA, USA. 4 p.

Technical Association for the Pulp and Paper Industries (TAPPI). 1998. Acidinsoluble lignin in wood and pulp. TAPPI Test Method T 222 om-98. Pechtree Corners, GA, USA. 7 p.

Wolcott, M. and K. Englund. 1999. A technology Review of Wood-Plastic Composites. In: Proceedings of the $33^{\text {rd }}$ International Particleboard/Composite Materials Symposium. Pullman, Washington, DC, USA. pp. 103-111.

Yuan, Q., D. Wu, J. Gotama and S. Bateman. 2008. Wood Fiber Reinforced Polyethylene and Polypropylene Composites with High Modulus and Impact Strength. Journal of Thermoplastic Composite Materials 21: 195-208.

Doi: $10.1177 / 0892705708089472$.

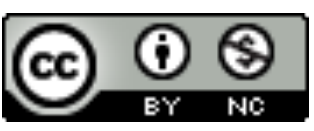

Todos los textos publicados por la Revista Mexicana de Ciencias Forestales -sin excepción- se distribuyen amparados bajo la licencia Creative Commons 4.0 Atribución-No Comercial (CC BY-NC 4.0 Internacional), que permite a terceros utilizar lo publicado siempre que mencionen la autoría del trabajo y a la primera publicación en esta revista. 